\title{
VERSIÓN PEDIÁTRICA DEL PROTOCOLO DE EVALUACIÓN DE LA ADECUACIÓN (PAE): APLICACIÓN A LOS CUATRO GRUPOS DIAGNÓSTICOS RELACIONADOS MÁS FRECUENTES EN UN HOSPITAL INFANTIL DE LA CORUÑA
}

\author{
Jesús Luis Saleta Canosa (1), Antonio Rodriguez Sotillo (2) y Arturo Aboal Seijas (3). \\ (1) Escuela Universitaria de Enfermería. Ferrol. \\ (2) Complejo hospitalario Juan Canalejo. La Coruña. \\ (3) Instituto Médico Quirúrgico San Rafael. La Coruña.
}

\section{RESUMEN}

Fundamento: Existe un amplio consenso en que una parte de la utilización de los recursos hospitalarios es inadecuada, en el sentido de que los pacientes reciben servicios que no les proporcionan beneficios significativos, o bien. que podrían ser más beneficiosos, o menos costosos, en un nivel asistencial inferior. El objetivo principal de este trabajo es determinar, mediante la aplicación retrospectiva de la versión pediátrica del Protocolo de Evaluación de la Adecuación (AEP), el porcentaje de ingresos y estancias inadecuadas de los 4 Grupos Relacionados con el Diagnóstico (GDR) más frecuentes en un hospital materno-infantil.

Método: Se trata de un estudio retrospectivo en el que se analizan 353 historias clínicas (HC) de las 361 que constituyen la población de estudio. Como análisis estadístico se utiliza Chi cuadrado y análisis de tendencia lineal. Sobre las medias obtenidas se aplicó un análisis de la varianza y la prueba de comparación múltiple de Scheffé.

Resultados: Las admisiones fueron juzgadis como adecuadas en $284(80.5 \%)$ de los casos e inadecuadas en $69(19,5 \%)$. El $76 \%$ de las estancias revisadas fueron juzgadas como apropiadas y el $24 \%$ como inapropiadas. En el GDR 030 (estupor y coma de origen traumático, coma<1 hora. edad $0-17$ ) se aprecia una relación directa entre duración de la estancia y uso inapropiado de la hospitalización.

Conclusiones: De los resultados de este estudio se desprende que una sustancial proporción de la utilización en el hospital es inapropiada y que, particularmente en el GDR 030, es probable se esté efectuando un manejo especialmente conservador.

Palabras clave: Pediátría. Frecuentación hospitalaria. Grupos Diagnósticos Relacionados.

Correspondencia:

Jesús Luis Saleta Canosa.

Antonio Ríos, $68.51 \mathrm{H}$.

15009 La Coruña.

\section{ABSTRACT \\ PaediatricVersion of the Adequacy \\ Evaluation Protocol (AEP): its \\ Application to the four most frequent \\ Diagnosis Related Groups in a Children's Hospital in La Coruña}

Background: There is wide-spread consensus that a part of the use of hospital resources is inadequate in the sense that the patients receive services that do not provide them with any significant benefits, or that could be more beneficial, or less costly, with a lower care standard. The main aim of this work is to determine the percentage of inadequate admissions and stays in hospital for the four most frequent Diagnosis-Related Groups (DRGs) in a maternity-children's hospital through the retrospective application of the paediatric version of the Adequacy Evaluation Protocol (AEP).

Method: It was a retrospective study in which 353 out of the 361 medical records that made up the covered population were analysed. Chi-square and linear trend were the statistical analysis methods used. A variance analysis and the Scheffé multiple comparison test were applied to the average values obtained.

Results: The admissions were judged to be adequate in 284 $(80.5 \%)$ of the cases and inadequate in $69(19.5 \%)$. Of the stays in hospital, $76 \%$ were deemed to be appropriate and $24 \%$ inappropriate. In DRG 030 (stupor and trauma-related coma, coma $<1$ hour, age 0-17), a direct relation can be found between the length of the stay in hospital and the inappropriate use of hospitalisation.

Conclusions: The results of this study show that a substantial proportion of the use of the hospital is inappropriate and that, particularly in DRG 030, this use is probably handled in an overly conservative way.

Key Words: Paediatrics. Number of times in hospital. Diagnosis-related Groups. 


\section{INTRODUCCIÓN}

Entre 1960 y 1991 el porcentaje del producto interior bruto (PIB) dedicado a gastos de salud en los países de la OCDE, salvo algunas excepciones, creció del 3,6 al 7,4\%. En España, durante este período se cuadruplicó la participación de la sanidad en el PIB, pasando de un 1,5 a un $6,7 \%^{1}$, suponiendo el componente hospitalario algo más del $60 \%$ del mismo. Aunque este gasto sea comparativamente menor que el de otros países desarrollados, la preocupación por su rápido crecimiento, la actual situación de recesión económica y las exigencias de la política de incorporación a la Unión Europea hacen previsible un futuro inmediato de austeridad para el sector sanitario.

El aumento del gasto sanitario puede describirse como resultado de: a) una continua elevación de precios, en general (inflación) y otra específica de cada subsector sanitario; b) una mayor densidad de pruebas diagnósticas y terapéuticas por proceso; c) una mayor utilización de los servicios (frecuentación hospitalaria, consumo de medicamentos, contactos con el médico), y d) el crecimiento de la población ${ }^{2}$.

Existe un amplio consenso en que parte de la utilización de los recursos hospitalarios es inadecuada, en el sentido de que los pacientes reciben servicios que no les proporcionan beneficios significativos, o bien, que podrían ser más beneficiosos o menos costosos, en un nivel asistencial inferior ${ }^{3.4}$. También es sabido que algunos de los días que los pacientes permanecen en el hospital, aún en el supuesto de que el internamiento en conjunto sea necesario, no se hallan en un estado clínico o no necesitan cuidados que requieran su estancia en el hospital durante ese día ${ }^{5,6}$.

Con la finalidad de determinar qué ingresos y estancias hospitalarias están o no justificadas se han desarrollado diversos instrumentos entre los que destaca el Protocolo de Evaluación de la Adecuación (AEP), cuya fiabilidad y validez ha sido demostra$\mathrm{da}^{7-9}$. Se dispone en la actualidad de dos versiones del AEP, una para aplicación a pacientes adultos y una versión para aplicación en pediatría.

El objetivo principal de este trabajo es determinar, mediante la aplicación retrospectiva de la versión pediátrica del AEP, el porcentaje de ingresos y estancias inadecuadas de los 4 Grupos Relacionados con el Diagnóstico $^{10,11}$ (GDR) más frecuentes en un hospital materno-infantil.

\section{MATERIAL Y MÉTODOS}

El estudio se llevó a cabo en el hospital materno-infantil Teresa Herrera, que forma parte del Complejo hospitalario Juan Canalejo -Marítimo de Oza de La Coruña, un hospital de nivel terciario ${ }^{12}$.

La población a estudio la constituyen todos los pacientes (361) encuadrados dentro de los 4 GDRs más frecuentes (tabla 1) ingresados en el período comprendido entre el 1 de julio de 1994 y 30 de junio de 1995.

Se trata de un estudio retrospectivo en el que se analizan 353 historias clínicas (HC) de las 361 que integran la población de estudio, ya que 8 de ellas $(2,2 \%)$ no se hallaron.

La unidad de análisis la constituye, por una parte el día de la admisión y, por otra. dos o más días de la estancia total del paciente (excepto la correspondiente al día del alta que no es revisada), ponderados según la duración de la misma (mínimo de 2 estancias evaluadas cuando la estancia total era inferior a 11 días y máximo de 4 días cuando dicha estancia total era superior a 20 días) y elegidos por muestreo aleatorio simple.

Los pacientes que permanecieron ingresados por un período igual o inferior a 24 horas sólo se valoraron para adecuación de ingreso pero no de estancia. 
Aunque la versión del AEP en pediatría se aplica a pacientes a partir de los 6 meses de edad ${ }^{13}$, arbitrariamente hemos incluido en nuestro estudio a pacientes con edad igual o superior a los 12 meses, por lo que la edad de los mismos se refleja en años cumplidos.

El tipo de ingreso del paciente se consideró urgente si se hacía a través del servicio de urgencias del hospital y programado si se hacía a través de consultas externas.

Como reingreso se interpretó todo aquel paciente que ingresa de nuevo en el hospital en los siguientes 30 días después de haber sido dado de alta a consecuencia de la misma patología (o complicaciones de la misma) que había originado el ingreso anterior.

La revisión fue realizada por un único observador, médico, con experiencia en revisión de $\mathrm{HC}$.

Para la identificación de admisiones y estancias inapropiadas se utilizó la versión pediátrica del AEP traducida al español y publicada por la Fundación Avedis Donabedian $^{13}$ (Tablas 2 y 3), formada por dos conjuntos de criterios objetivos e independientes del diagnóstico, destinados a la identificación de admisiones y estancias inapropiadas.

El primer conjunto consta de 20 criterios diseñados para juzgar la necesidad de la admisión hospitalaria. Los siete primeros tienen relación con los servicios clínicos que recibe el paciente, mientras que los 13 restantes con la gravedad del estado clínico del paciente. La presencia durante el primer día de ingreso hospitalario de uno solo de estos criterios basta para considerar apropiado el ingreso, que se valora como inapropiado cuando no cumpla ninguno de ellos. Es importante destacar que este primer grupo de criterios valora la pertinencia de la admisión en el momento en que ésta se produce y no la totalidad del ingreso hospitalario.
El segundo conjunto de criterios se empleó para determinar la necesidad de cada una de las estancias siguientes al día del ingreso, con la excepción, como ya quedó señalado, del día del alta. Consta de un total de 28 criterios, 12 de ellos referidos a la prestación de servicios médicos, 8 a cuidados de enfermería y los 8 últimos a la situación clínica del paciente. Como en el conjunto anterior, basta con presentar un criterio para considerar apropiada la estancia hospitalaria el día revisado.

A diferencia de algunos trabajos publica$\operatorname{dos}^{14}$, en el que se ha cambiado alguno de los ítems, no se realizó modificación alguna.

El AEP incorpora, además una lista de posibles motivos de uso innecesario (tablas 4 y 5), que facilita el poder asignar a la admisión y a los días de estancia, considerados como inadecuados, una posible causa. Dado que los items utilizados son los mismos que se aplican al AEP de adultos, algunos de ellos no son de aplicación en pediatría, tales como el ítem n 17 (el paciente precisa una residencia de ancianos) y otros, se modificaron para su mejor adaptación a un contexto pediátrico (cambiando la palabra «anciano» por «niño»).

Previa elaboración de una ficha epidemiológica de recogida de datos, figura 1 , se solicitaron las $361 \mathrm{HC}$ una sola vez, al archivo del hospital. Un total de 19 pacientes $(5,36 \%)$ ingresaron por un período igual o inferior a 24 horas, por lo que sólo se valoraron para la adecuación de la admisión pero no de la estancia. Los restantes 334 casos originaron 2085 estancias.

Todos los documentos contenidos en la $\mathrm{HC}$ estuvieron disponibles y se utilizaron en la revisión.

Para el análisis de los datos se creó una base de datos en el programa informático dBASEIII. Los datos se analizaron con los paquetes estadísticos EPIINFO y SPSS para Windows. Sobre variables cuantitativas se calculó la media y desviación estándar, así 
como los valores máximos y mínimos. Como análisis estadístico se utiliza Ji cuadrado y análisis de tendencia lineal. Sobre las medias obtenidas se aplicó un análisis de la varianza y para detectar las diferencias entre ellas se aplicó la prueba de comparación múltiple de Scheffé.

\section{RESIJITADOS}

La edad media de los pacientes fue $7,71 \pm 3,65$ (1-16) años. Sólo 2 pacientes tenían edad superior a 13 años . En cuanto al sexo, $218(61,8 \%)$ eran varones y 135 $(38,2 \%)$ niñas.

Los ingresos procedentes del servicio de urgencias fueron $347(98,3 \%)$, mientras que sólo $6(1,7 \%)$ se realizaron de forma programada. La estancia media fue 6,32 $\pm 4,61$ (241) días, sin contar el día del alta. Dicha estancia media fue mayor en el GDR 026

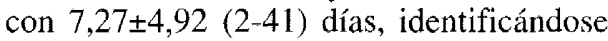
diferencias estadísticamente significativas entre éste y el GDR $030(\mathrm{p}=0,001)$. En la tabla 6 se muestran las características de la población estudiada y tipo de ingreso por GDR. Sólo 2 pacientes permanecieron ingresados por un período superior a 30 días. Ningún paciente falleció. Únicamente se observaron $2(0,6 \%)$ readmisiones.

Las admisiones fueron juzgadas como adecuadas en $284(80,5 \%)$ de los casos e inadecuadas en $69(19,5 \%)$. Los porcentajes de inadecuación de admisiones por GDR se presentan en la figura 2, encontrándose diferencias estadísticamente significativas entre todos ellos $(\mathrm{p}<0,003)$, excepto entre el GDR 026 y GDR 070.

De las 2085 estancias que originaron los 334 pacientes, se estudiaron $684(38,8 \%)$. El $76 \%$ de los días revisados fueron juzgados como necesarios y el $24 \%$ como innecesarios. En la figura 3 quedan reflejados los porcentajes de inadecuación de estancias por GDR. Las diferencias fueron estadísticamente significativas $(p<0,003)$, ex- cepto cuando se compara el GDR 070 con el GDR 184.

No se encontraron diferencias significativas entre las estancias juzgadas como innecesarias y duración de la misma cuando los datos se analizan de forma global $(p=0,94)$. Sin embargo, en el GDR 030, los pacientes con estancias menores de 11 días (grupo de referencia) mostraron diferencias estadísticamente significativas $(p=0,01)$ cuando se comparan con los que tuvieron estancias entre 11 y 20 días y más de 20 días.

Tampoco hubo relación entre estancias innecesarias y sexo del paciente cuando los datos se estudian desde una perspectiva global $(\mathrm{p}=0,06)$. No obstante, al evaluar dicha asociación en los distintos GDR, ésta se pone de manifiesto en el GDR $026(\mathrm{p}=0,04)$.

Cuando se comparan las estancias juzgadas como innecesarias en el grupo de pacientes con 5 o menos años de edad respecto a los de más de edad, tampoco se encuentran diferencias significativas $(p=0,281)$.

Aquellos casos en los que el ingreso se catalogó como inadecuado, se identificaron todos ellos como debidos a pruebas diagnósticas y/o tratamiento que hubieran podido realizarse en consultas externas.

Los motivos de inadecuación de las estancias se atribuyeron en el $71,4 \%$ de los casos a que el médico «no presta atención a la necesidad de dar el alta rápidamente, una vez que se ha conseguido el propósito de la hospitalización: el médico mantiene al paciente en el centro cuando ya no recibe o requiere los servicios de un centro de agudos». En el $20,5 \%$ se debió a que el procedimiento diagnóstico y/o tratamiento necesario pudiera haberse realizado como paciente externo; en el $5,4 \%$ a que se estaba pendiente de resultados de prueba(s) diagnóstica(s) o interconsultas para la toma de decisiones terapéuticas y/o diagnósticas. Mcnos del 3\% fucron debidas a otras causas. 


\section{DISCUSIÓN}

De los resultados de este estudio se desprende que una elevada proporción de la utilización hospitalaria es inapropiada, aunque los resultados de este trabajo deben tomarse con cierta cautela dado que se utiliza un protocolo (AEP pediátrico) que aún no está suficientemente validado en niños, aunque sí en adultos.

El porcentaje de admisiones e ingresos inapropiados es más alto que el encontrado en algunos estudios ${ }^{15}$ e idéntico al publicado en otros ${ }^{16}$, realizados también en pacientes de pediatría, si bien los resultados no son del todo comparables dado que en este trabajo se analizan 4 GDRs y en la bibliografía consultada se muestrea todo tipo de patologías (servicios). Como en otros estudios ${ }^{17.18}$, la razón que identifica la mayor parte de las estancias juzgadas como innecesarias fue un manejo médico conservador. Esto adquiere especial importancia en tanto en cuanto esta causa de inadecuación depende del comportamiento del médico y por lo tanto es susceptible de ser modificada (establecimiento de protocolos, informar al clínico acerca de los resultados del AEP, etc.).

La única causa de ingreso inadecuado se identificó como debida a pruebas diagnósticas y/o tratamientos que hubieran podido efectuarse en consultas externas. Este motivo de inadecuación viene a coincidir con otros ya publicados en la literatura ${ }^{19}$. El mejorar la accesibilidad a la realización de pruebas diagnósticas y muy especialmente en cuanto a su rapidez se refiere, probablemente determine una importante disminución de ingresos innecesarios.

Los datos en la literatura acerca de la relación entre duración de la estancia y uso inapropiado de la hospitalización son discordantes ${ }^{8.14 .16 .18}$. Dicha asociación la presenta en nuestro estudio el GDR 030. Este hallazgo nos induce a pensar en la posibilidad de que se esté haciendo un manejo especialmente conservador en el traumatismo craneal o craneoencefálico, sobre todo si se tiene en cuenta que la razón de uso inapropiado en este GDR se atribuyó en el $76 \%$ de los casos a este motivo.

No encontramos una razón en el GDR 026, que explique el mayor porcentaje de uso inapropiado de la hospitalización en niñas. Otros estudios sobre uso inapropiado realizados en pacientes adultos ${ }^{20}$ informan también acerca de esa asociación.

Un instrumento de medida del uso innecesario de la hospitalización como el AEP tiene una serie de limitaciones ${ }^{21}$ que es importante resaltar: 1) se asume que todos los cuidados prestados son siempre apropiados desde el punto de vista clínico, con independencia del nivel donde se prestan y el momento o tiempo de su presentación; 2) también se asume que los datos básicos para determinar la necesidad médica de la hospitalización se hallan documentados en la historia clínica; 3 ) interpreta que sólo las consideraciones clínicas y algunos factores sociales que afectan a la evolución clínica, justifican la prestación de cuidados bajo ingreso hospitalario. Otros factores, como la inexistencia de un soporte social suficiente, la distancia que tenga que recorrer el paciente para recibir servicios ambulatorios, etc, no se consideran justificantes clínicos de la hospitalización. Es en este sentido que la palabra «innecesario» puede reflejar la falta de alternativas para esos pacientes y familiares, sin olvidar que la hospitalización tiene un impacto económico así como emocional e incluso riesgo físico (infección nosocomial) para el paciente.

La utilización de métodos de identificación del uso inapropiado como el AEP puede tener aplicaciones diversas tanto en planificación como en gestión hospitalaria, al permitir identificar problemas hospitalarios causantes de demoras, principalmente problemas de tipo organizativo, permitiendo el desarrollo de intervenciones encaminadas a la reducción del uso inapropiado. De hecho, algunos estudios han mostrado que la retroinformación del uso in- 
necesario a clínicos y gestores se ha mostrado eficaz para conseguir esa reducción ${ }^{22,23}$. La detección de pacientes ingresados que no requieren hospitalización y la identificación de los principales motivos del mismo, por ejemplo excesiva distancia al hospital de pacientes en rehabilitación que tienen que acudir al hospital durante varios días, necesidad de tener que realizar un diagnóstico rápido, entre otros, permitirán plantearse la necesidad de realizar modificaciones en los sistemas de organización hospitalaria para poder realizar pruebas ambulatorias en plazos más razonables o cambios organizativos como la creación de hoteles para pacientes, como el que en la actualidad funciona en el complejo hospitalario Juan Canalejo, que pueden dar una nueva perspectiva a la solución de estos problemas.

La mejora de la calidad asistencial como consecuencia no sólo de una menor probabilidad de infección nosocomial, al reducir el número de estancias innecesarias, sino también de una reducción en las listas de espera y niveles de ocupación, pueden ser otras de las consecuencias derivadas de la utilización del AEP.

Por último, resaltar que, si bien son yà numerosos los trabajos publicados en nuestro país sobre la aplicación del AEP en adultos, cuya validación en nuestro entorno ya ha sido demostrada ${ }^{y}$, no ocurre así con la aplicación del AEP pediátrico, ya que los estudios publicados son sensiblemente inferiores en número y, lo que es más importante, su validación queda todavía pendiente de comprobación.

\section{BIBLIOGRAFÍA}

1. Puig Junoy J. Gasto sanitario y políticas de contención de costes: Hechos y tendencias recientes en el caso español. 20 ed. Barcelona. Rev Economía Aplicada 1994.

2. Ortún Rubio v. La Economía en Sanidad y Medicina: Instrumentos y limitaciones. 10 ed. Barcelona: Escola Universitaria de Treball Social: La Llar del Llibre, S.A.; 1991.

3. Fuente D, Peiró S, Portella E, Marchan C., Aymerich $\mathrm{S}$. Utilización innecesaria de la hospitaliza- ción: Importancia de la gestión a nivel de Servicio. Rev Calidad Asistencial 1994; 1: 8-16.

4. Peiró S, Meneu de Guillena R, Roselló ML, Martínez E, Portella E. QuÉ mide la estancia media de los grupos relacionados de diagnóstico? Med Clin (Barc) 1994; 103: 413-417.

5. Mozes B, Katz A, Halkin H, Schiff and Modan B. Reduction of redundant hospital stay through controlled intervention. Lancet 1987; 968-970.

6. Winickoff R, Restuccia JD and Fincke B. Concurrent Application of the Appropriateness Evaluation Protocol to Acute Admissions in Department of Veterans Aftairs Medical Centers. Med Care 1991; 29 (8), supplement.

7. Gertman PM and Restuccia JD. The Appropiateness Evaluation Protocol: A technique for assessing unnecesary days of hospital care: Med Care $1981 ; 19 ; 855-870$.

8. Canga E, Del Vigo S, Fabo P, Sañudo S, Lopez O. Experiencia en la aplicación del «The Appropiateness Evaluation Protocols: Evaluación de método. Todo Hosp 1994; 103: 29-34.

9. Peiró S, Meneu R, Martinez E, Portella E. Validez de un método de identificación del uso innecesario de la hospitalización. V Congreso de la Sociedad Española de Salud Pública y Administración Sanitaria. Granada; 1993.

10. Sistema Nacional de Salud año 1994: Explotación de bases del CMBD. Estadísticos de referencia estatal de los sistemas de agrupación de registros de pacientes (G.R.D. y P.M.C.). Madrid: Ministerio de Sanidad y Consumo; 1996.

11. CIE $9 \mathrm{MC}$ : Clasificación Internacional de Enfermedades, 9? Revisión. Modificación Clínica. Volumen 1: Enfermedades Lista Tabular. 20 ed. Mar drid: Ministerio de Sanidad y Consumo; 1994.

12. Resolución 11-4-1980. Secretaría de Estado para la Sanidad. BOE núm. 107 de 3-5-1980.

13. Restuccia JD. Appropiateness Evaluation Protocol: Manual para la revisión. Barcelona: Fundació Avedis Donabedian; 1996.

14. Kemper KJ. Medically Inappropiate Hospital use in a Pediatric Population. N Eng J Med 1988; 318 (16): 1033-1037.

15. Kreger BE, Restuccia JD. Assessing the need to hospitalize children: pediatric appropriateness evaluation protocol. Pediatrics $1989 ; 84$ (1): 242 247.

16. Gloor JE, Kissoon N, Joubert GI. Appropriateness of hospitalization in a Canadian pediatric hospital. Pediatrics 1993; 91: 70-74. 
17. Booth BM, Ludke RL, Wakefield DS, Kern DC, Burmeister LF, Fisher EM, et al. Nonacute days of care Within department of veterans affairs medical centers. Med Care 1991; 29 (suppl): AS51AS63.

18. Restuccia JD, Holloway DC. Barriers to appropriate utilization of an acute facility. Med Care 1976; 14: 559-573.

19. Bañeres J, Alonso J, Broquetas J, Antó JM. Ingresos hospitalarios inadecuados y días de estancia inactivos en pacientes con enfermedad pulmonar obstructiva crónica y neoplasia pulmonar. Med Clin (Barc) 1993: 100: 407-411.
20. Payne SMC, Restuccia JD, Ash A et al. Using utilization review information to improve hospital efficiency. Hosp Health Serv Administration 1991; 36: 473-490.

21. Peiró S, Portella E. Identificación del uso inapropiado de la hospitalización: la búsqueda de la ineficiencia. Med Clin (Barc) 1994; 103 (2): 65-71.

22. Restuccia JD. The effect of concurrent feedback in reducing inappropriate hospital utilization. Med Care 1982; 20: 46-62.

23. Payne SMC, Ash A, Restuccia JD. The Role of Feedback in Reducing Medically Unnecesary Hospital Use. Med Care 1991; 29 (suppl): 91-105. 


\section{FE DE ERRATAS}

En el número 3 de 1997 de la Revista Española de Salud Pública se publicó el trabajo: Versión pediátrica del protocolo de evaluación de la adecuación (PAE): Aplicación a los cuatro Grupos Diagnósticos relacionados más frecuentes en un hospital infantil de la Coruña». Saleta Canosa JL, Rodríguez Sotillo A y Aboal Seijas A. Rev Esp Salud Pública 71(3): 249-255. En la publicación no figuraban las tablas y figuras que se citaban en el texto y que se reproducen a continuación.

Tabla 1

Grupos relacionados con el diagnóstico más frecuente

\begin{tabular}{|clcc|}
\hline GRD & \multicolumn{1}{c}{ Descripción } & N. ${ }^{\circ}$ casos & $\%$ \\
\hline 030 & Estupor y coma de origen traumático, comá < 1 hora, edad $0-17$ & 110 & 30.47 \\
026 & Convulsiones y cefalea, edad 0-17 & 100 & 27,7 \\
070 & Otitis media y otras infecciones ORL, excepto laringotraqueitis, edad 0-17 & 77 & $21,3,3$ \\
184 & Esofagitis, gastroenteritis y otras enf. digestivas, edad 0-17 & 74 & 20,5 \\
\hline & TOTAL & 361 & 100 \\
\hline
\end{tabular}


Tabla 2

Adecuación del ingreso

\section{SERVICIOS CLINICOS}

1. Cirugía o Técnica especial en 24 h. que requiera.

- anestesia general o regional

- equipamiento o medios disponibles sólo en ingresos

2. Tratamiento en una UCI.

3. Telemetría o monitorización de constantes vitales cada $2 \mathrm{~h}$.

4. Medicación intravenosa y/o reposición de fluidos

(No se incluye alimentación por sonda)

5. Observación de reacción secundaria no deseada a medicación

6. Antibióticos intramusculares al menos cada 8 horas

7. Ventilación asistida continua o intermitente (al menos cada 8 h.)

\begin{tabular}{|c|c|c|c|}
\hline \multicolumn{4}{|c|}{ SITUACION CLINICA DEL PACIENTE } \\
\hline 8. & \multicolumn{3}{|c|}{$\begin{array}{l}\text { Alteración electrolítica/ácido-base severa: } \\
\text { - } \mathrm{Na}<123 \mathrm{mEq} / \mathrm{l} \text { o }>156 \mathrm{mEq} / \mathrm{l} \\
-\mathrm{K}<2,5 \mathrm{mEq} / \mathrm{l} \text { o }>6 \mathrm{mEq} / \mathrm{l} \\
\text { - } \mathrm{HCO} 3<20 \mathrm{mEq} / \mathrm{l} \circ>36 \mathrm{mEq} / \mathrm{l} \\
-\mathrm{pH} \text { arterial }<7.3 \circ>7,45\end{array}$} \\
\hline 9. & \multicolumn{3}{|c|}{ Ficbre persistente $>38^{\circ} \mathrm{C}$ durante más de 5 días } \\
\hline 10. & \multicolumn{3}{|c|}{ Pérdida brusca de movilidad corporal (déficit motor) } \\
\hline 11. & \multicolumn{3}{|c|}{ Pérdida brusca de visión o audición } \\
\hline 12. & \multicolumn{3}{|c|}{ Hemorragia activa } \\
\hline 13. & \multicolumn{3}{|c|}{ Evisceración o dehiscencia de herida quirúrgica } \\
\hline \multirow[t]{5}{*}{14.} & \multicolumn{3}{|c|}{ Pulso arterial fuera de los rangas siguientes (óptimo, paciente de 12 años durmiendo): } \\
\hline & - 6-23 meses & $80-200 \mathrm{ppm}$ & \\
\hline & - 2-6 años & $70-200 \mathrm{ppm}$ & \\
\hline & - 7-11 años & $60-180 \mathrm{ppm}$ & \\
\hline & - 12 años & $50-140 \mathrm{ppm}$ & \\
\hline \multirow[t]{6}{*}{15 . } & \multicolumn{3}{|c|}{ Presión arterial fuera de los rangos: } \\
\hline & & sistólica & diastólica \\
\hline & - 6-23 meses & $70-120 \mathrm{~mm} \mathrm{Hg}$ & $40-85 \mathrm{~mm} \mathrm{HG}$ \\
\hline & - 2-6 años & $75-125 \mathrm{~mm} \mathrm{Hg}$ & $40-90 \mathrm{~mm} \mathrm{HG}$ \\
\hline & - 7-11 años & $80-130 \mathrm{~mm} \mathrm{Hg}$ & 45-90 mm HG \\
\hline & - 12 años & $90-200 \mathrm{~mm} \mathrm{Hg}$ & $60-120 \mathrm{~mm}$ HG \\
\hline 16. & \multicolumn{3}{|c|}{ Estado confusional agudo, coma o falta de respuesta } \\
\hline 17. & \multicolumn{3}{|c|}{ Hematocrito $<30 \%$} \\
\hline 18. & \multicolumn{3}{|c|}{ Necesidad de punción lumbar, cuando este procedimiento no se realice de forma rutinaria en consultas externas } \\
\hline 19. & \multicolumn{3}{|c|}{ Entidades en las que el paciente no responda a tratamiento como paciente externo } \\
\hline 20. & $\begin{array}{l}\text { Problemas ped } \\
\text { - Abusos } \\
\text { - Incumplimic } \\
\text { - Necesidad d } \\
\text { se sospeche) }\end{array}$ & $\begin{array}{l}\text { monio } \\
\text { monitorización d }\end{array}$ & Iuyendo ingesta calórica (aunque sólo \\
\hline
\end{tabular}


Tabla 3

Adecuación de la estancia pediátrica

\begin{tabular}{|rl|}
\hline \multicolumn{1}{|c|}{ SERVICIOS MÉDICOS } \\
\hline 1. & Intervención quirúrgica ese mismo día \\
2. & Intervención quirúrgica en 24 h (necesaria evaluación preoperatoria) \\
3. & Cateterización cardíaca ese mismo día \\
4. & Angiografía ese mismo día \\
5. & Biopsia de órgano interno ese mismo día \\
6. & Toracocentesis o paracentesis cse mismo día \\
7. & Procedimicnto invasivo en SNC ese mismo día \\
8. & Endoscopia gastrointestinal ese mismo día \\
9. & Cualquier prueba con control dietético estricto \\
10. & Tratamiento nuevo que requiera frecuentes ajustes de dosis \\
11. & Control monitorizado al menos 3 veces/día \\
12. & Procedimiento invasivo durante las últimas 24 h. \\
\hline
\end{tabular}

\begin{tabular}{|ll|}
\hline \multicolumn{1}{|c|}{ CUIDADOS DE ENFERMERÍA } \\
\hline 13. & Ventilación mecánica y/o terapia respiratoriá por inhalación al menos 3 veces al día \\
14. & Terapia parental intermitente o continua \\
15. & Monitorización de constantes al menos cada $30 \mathrm{~min} .4 \mathrm{~h} . \mathrm{mínimo})$ \\
16. & Inyecciones intramusculares o subcutáneas al menos 2 veces al día \\
17. & Control de balances \\
18. & Cuidado de heridas quirúrgicas mayores y drenajes \\
19. & Tracción de fracturas, luxaciones o deformidades congénitas \\
20. & Moniorización por una enfermera al menos 3 veces/día (con supervisión médica) \\
\hline
\end{tabular}

\section{SITUACIÓN CLÍNICA DEL PACIENTE}

Durante el mismo dia o 24 h. antes:

21. Ausencia de motilidad intestinal o incapacidad para orinar

\section{Dentro de las $48 h$. anteriores:}

22. Fiebre de al menos $38^{\circ} \mathrm{C}$

23. Trasfusión debida a pérdida de sangre

24. Fibrilación ventricular o ECG de isquemia aguda

25. Coma-pérdida de conocimiento durante $1 \mathbf{h}$.

26. Estado confusional agudo (excluyendo síndrome de abstinencia alcohólica)

27. Síntomas o signos debidos a perturbación hematológica aguda

28. Dificultades neurologicas 
Tabla 4

\section{INGRESO INADECUADO}

1. Pruebas diagnósticas y/o trataniento pueden realizarse en consultas externas

2. El paciente ha sido ingresado para realizar pruebas diagnósticas y/o tratamiento pueden realizarse en consultas externas. excepto si el paciente vive muy lejos como para efectuarse de forma rápida

3. Pruebas diagnósticas y/o tratamiento pueden realizarse en consultas externas. excepto si el paciente no está ingresado

4. El paciente requiere institucionalización, pero a un nivel inferior (no especificado), no en un hospital de agudos

5. El paciente requiere cuidados en un hospital de crónicos

6. El paciente requiere cuidados de enfermería

7. El paciente precisa una residencia de ancianos

8. Admisión prematura-un día o más previos a la citación de las pruebas

9. Tratamiento o pruebas diagnósticas no documentados

10. Procedimiento quiruirgico que debería ser realizado de forma ambulatoria

11. El paciente precisa cuidados terminales

12. Abuso (o sospecha) del niño, paciente ingresado para custodia

13. Incumplimiento del tratamiento necesario

19. Otras (especificar)

Tabla 5

Día de estancia inadecuado

\section{Retraso en el desarrollo del estudio o tratamiento para el que el paciente está hospitalizado}

20. Problema de calendario para realizar intervención quirúrgica

21. Problema de calendario para realizar pruebas diagnósticas

22. Ingreso prematuro

23. Paciente cilado para prueba diagnóstica o tratamiento (incluyendo cirugía) cuya cita es anulada. por cualquier razón (caso urgente se antepone a caso electivo, personal crucial del centro está enfermo, etc.)

24. «Días de no trabajo» en el hospital: determinados procedimientos diagnósticos no se realizan durante los fines de semana ni en vacaciones

25. Pendiente de resultados de prueba(s) diagnóstica(s) o interconsulta(s) para la toma de decisiones terapéuticas y/o diagnósticas

29. Otras (especificar)

\begin{tabular}{|c|c|}
\hline \multicolumn{2}{|r|}{ Responsabilidad del médico o del hospital } \\
\hline 30. & Alta planeada pero no hay ódenes escritas \\
\hline 31. & Fallo en la hoja al iniciar/ejecutar el alta hospitalaria \\
\hline 32. & $\begin{array}{l}\text { No se presta atención a la necsidad de dar el alta rápidamente, una wey yue se ha conseguido el propésito de la } \\
\text { hospitalisación: el médico mantiene al paciente en el centro cuando ya no recibe o tequiere los servicios de un centro } \\
\text { de agudos }\end{array}$ \\
\hline 33. & No existe ningún plan de diagnóstico y/o tratamiento \\
\hline 34. & Cetalyuier procedimiento diagnistico $y /$ o tratamiento necesario puede realizarse como paciente externo \\
\hline 39 . & Otras (especificar) \\
\hline
\end{tabular}


Tabla 5 (continuación)

\section{Responsabilidad del médico o de la familia del paciente}

40. Falta de familiares para atención al paciente en su domicilio

11. Falta de preparación de la familia para atención del paciente en su domicilio

42. El paciente/la familia rechazan los medios disponibles en instalaciones alternativas

49. Otras (especificar)

\section{Responsabilidad del medio}

50. Paciente perteneciente que proviene de un ámbito social degradado, permanece ingresado en el hospital hasta que su ambiente se hace aceptable o se encuentra una opción alternativa

51. Paciente convaleciente de una enfermedad y se prevee que sólo se dispone de una estancia de menos de 72 h. en instalaciones alternativas

52. No disponibilidad de una instalación alternativa

53. No disponibilidad de tratamiento altemativo, del tipo de la altención domiciliatia

59. Otras (especificar)

Tabla 6

Características de la población y tipo de ingreso por GRD

\begin{tabular}{|c|c|c|c|c|c|}
\hline & $G R D O 30$ & GRIMISh & GRDO70 & GRDI84 & TOTAL \\
\hline N. ${ }^{\circ}$ pacientes & 111 & $9 \%$ & 76 & 70 & 353 \\
\hline Edad media & 7.9 & $\therefore 11$ & 6.9 & 7,2 & 7.6 \\
\hline Estancia media & 5.2 & 7,3 & 6,4 & 6,7 & 6.3 \\
\hline \multicolumn{6}{|l|}{ Sexo } \\
\hline Varón & $76(68.5 \%)$ & $59(61,5 \%)$ & $47(61,8 \%)$ & $36(51,4 \%)$ & $218(61.8 \%)$ \\
\hline Mujer & $35(31.5 \%)$ & $37(38,5 \%)$ & $29(38.2 \%)$ & $34(48,6 \%)$ & $135(38,2 \%)$ \\
\hline \multicolumn{6}{|l|}{ Tipo de ingreso } \\
\hline Urgente & $111(100 \%)$ & $93(96,9 \%)$ & $76(100 \%)$ & $67(95.7 \%)$ & $347(98,3 \%)$ \\
\hline Programado & $0(0 \%)$ & $3(3,1 \%)$ & $0(0 \%)$ & $3(4.3 \%)$ & $6(1,7 \%)$ \\
\hline Reingreso & $2(1,8 \%)$ & 0 & 0 & 0 & $2(0,6 \%)$ \\
\hline
\end{tabular}

* Se calcula sobre los pacientes que permacieron ingresados más de 24 horas. 


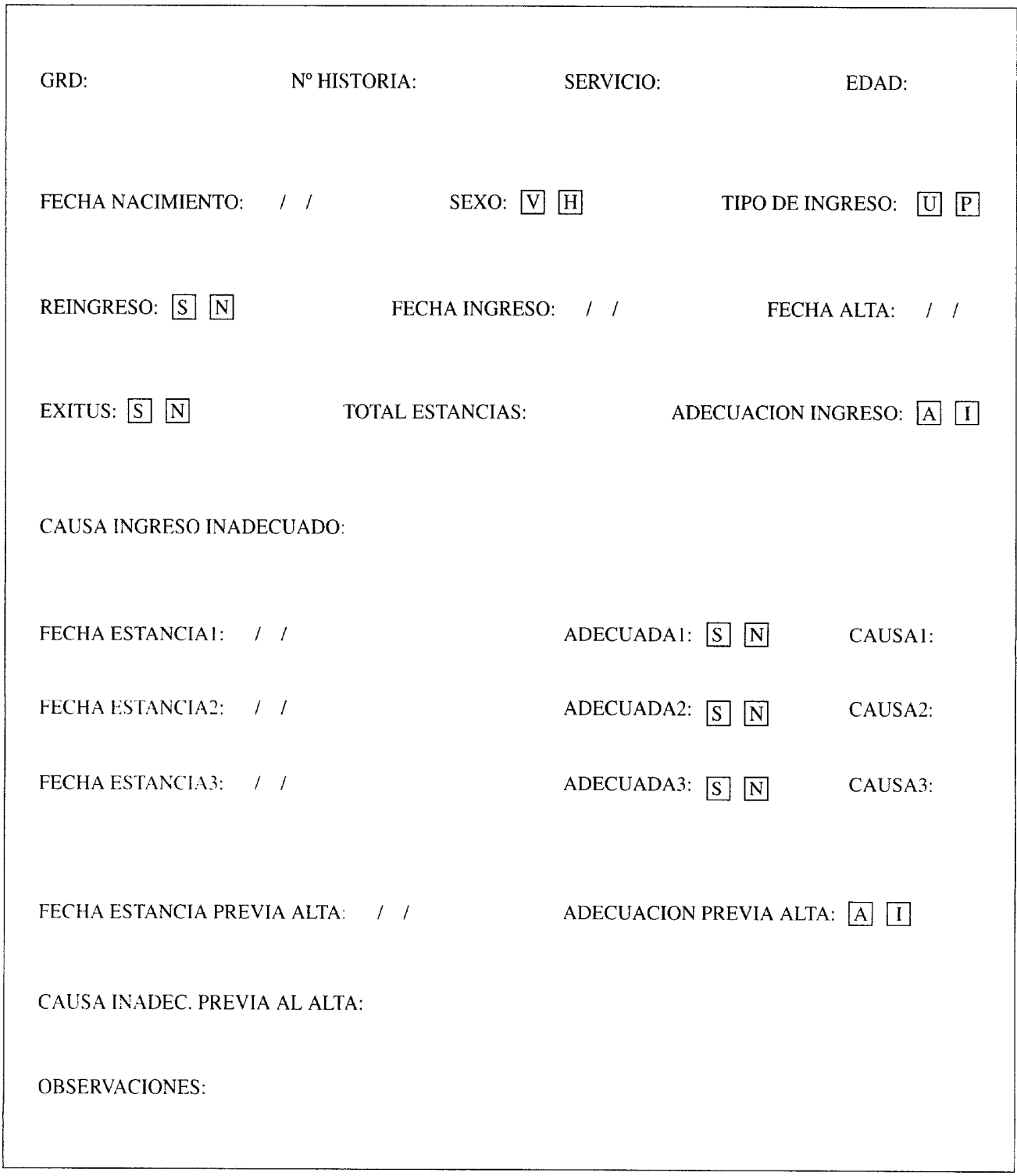


Figura 2

Porcentaje de ingresos inadecuados por GRD
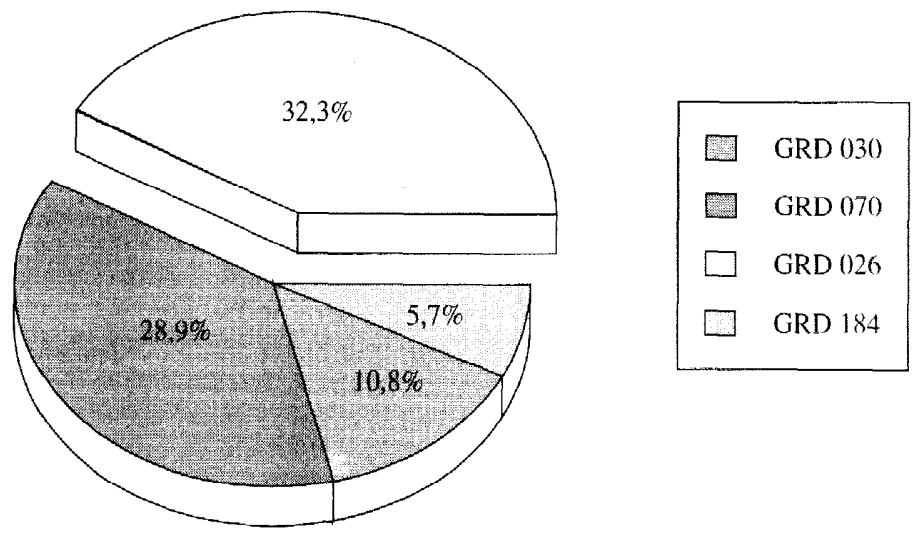

Figura 3

Porcentaje de estancias inadecuadas por GRD

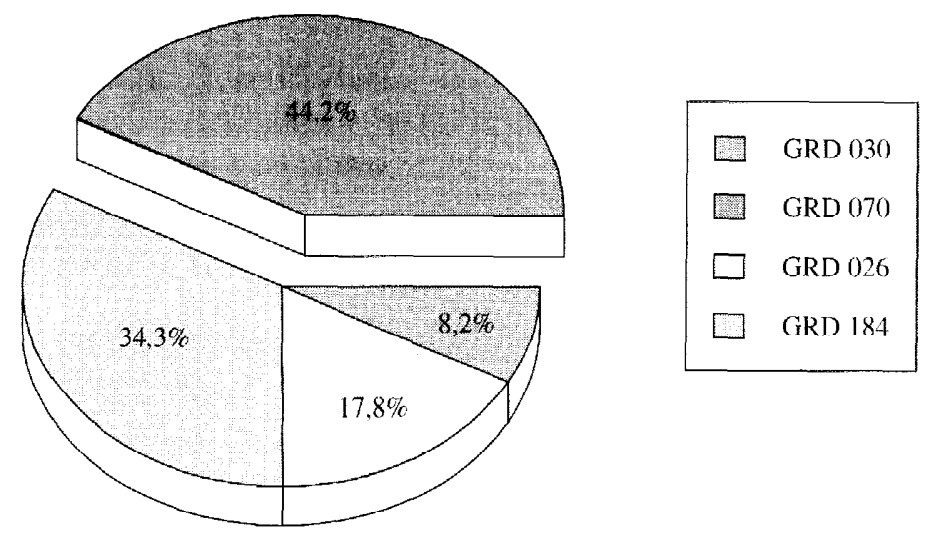

